\title{
COMPUTATION OF CONFORMAL REPRESENTATIONS OF COMPACT RIEMANN SURFACES
}

\author{
GUILLERMO LÓPEZ LAGOMASINO, DOMINGO PESTANA, JOSÉ M. RODRÍGUEZ, \\ AND DMITRY YAKUBOVICH
}

\begin{abstract}
We find a system of two polynomial equations in two unknowns, whose solution allows us to give an explicit expression of the conformal representation of a simply connected three-sheeted compact Riemann surface onto the extended complex plane. This function appears in the description of the ratio asymptotic of multiple orthogonal polynomials with respect to so-called Nikishin systems of two measures.
\end{abstract}

\section{INTRODUCTION}

Conformal representations of compact Riemann surfaces play an essential role in approximation theory; in particular, they serve to describe the so-called ratio asymptotic of orthogonal polynomials on the real line.

Let $\mu$ be a finite positive Borel measure whose compact $\operatorname{support} \operatorname{supp}(\mu)$ has infinitely many points and is contained in the real line. By $q_{n}(w)=\kappa_{n} w^{n}+\cdots+\kappa_{0}$, with $\kappa_{n}>0$, we denote the $n$th orthonormal polynonomial with respect to $\mu$; that is,

$$
\int q_{n}(t) q_{m}(t) d \mu(t)=\delta_{n, m},
$$

where $\delta_{n, m}$ denotes the Kronecker delta.

It is well known and easy to verify that the sequence $\left\{q_{n}\right\}, n \geq 0$, satisfies a three-term recurrence relation

$$
t q_{n}(t)=a_{n+1} q_{n+1}(t)+b_{n} q_{n}(t)+a_{n} q_{n-1}(t), \quad n \geq 1,
$$

$b_{n} \in \mathbb{R}, a_{n}>0$. The following result establishes a close connection between the ratio asymptotic of orthonormal polynomials and the limit behavior of the recurrence coefficients (see [6]).

Theorem 1.1. The following assertions are equivalent:

Received by the editor October 21, 2008 and, in revised form, February 13, 2009.

2000 Mathematics Subject Classification. Primary 30F99; Secondary 05E35, 30C30, 58C15.

Key words and phrases. Orthogonal polynomials, compact Riemann surfaces, branched covering, nonlinear equations, Newtonian continuation method.

The first, second, and third authors' research was partially supported by a grant from M.E.C. (MTM 2006-13000-C03-02) and a grant from U.C.III M./C.A.M. (CCG07-UC3M/ESP-3339), Spain.

The second and third authors' research was partially supported by two grants from M.E.C. (MTM 2006-11976 and MTM 2007-30904-E), Spain.

The fourth author's research was partially supported by the Grant MTM2008-06621-C02-01, DGI-FEDER, of the Ministry of Science and Innovation, Spain.

(C)2009 American Mathematical Society Reverts to public domain 28 years from publication 
- $\lim _{n} a_{n}=a>0, \lim _{n} b_{n}=b$.

- $\lim _{n} \frac{q_{n+1}(w)}{q_{n}(w)}=\varphi(w):=\frac{w-b}{2 a}+\sqrt{\left(\frac{w-b}{2 a}\right)^{2}-1}$, uniformly on each compact subset of $\mathbb{C} \backslash \operatorname{supp}(\mu)$.

If any of these conditions holds, then $\operatorname{supp}(\mu)=[b-2 a, b+2 a] \cup e$, where $e$ is at most a denumerable set of isolated points in $\overline{\mathbb{R}} \backslash[b-2 a, b+2 a]$.

The function $z=\varphi(w)$ is the conformal representation of $\overline{\mathbb{C}} \backslash[b-2 a, b+2 a]$ onto the complement of the unit disk such that $\varphi(\infty)=\infty, \varphi^{\prime}(\infty)>0(\overline{\mathbb{C}}$ denotes the extended complex plane). It is a solution of the algebraic equation

$$
z^{2}-\frac{w-b}{a} z+1=0
$$

which lives on a two-sheeted compact Riemann surface. The numbers $a$ and $b$ are determined by the first two coefficients of the Laurent expansion of $\varphi$ at $\infty$; in fact, $\varphi(w)=w / a-b / a+\mathcal{O}(1 / w), w \rightarrow \infty$. In turn, the algebraic equation which characterizes $\varphi$ is easy to determine if the values $a, b$ are given. Any measure for which $\operatorname{supp}(\mu)=[b-2 a, b+2 a] \cup e$, where $e$ is as described in the theorem, verifies this ratio asymptotic if $\mu^{\prime}>0$ a.e. on $[b-2 a, b+2 a]$. This is known as the Rakhmanov-Denisov theorem (see [3] and [9]).

Recently (see [1, 2], and [5]), results analogous to those stated above and the Denisov-Rakhmanov theorem were obtained for multiple orthogonal polynomials of Nikishin systems of $m$ measures. In this case, the ratio asymptotic is described in terms of a conformal representation of an $(m+1)$-sheeted compact Riemann surface onto the extended complex plane. The reader interested in these results can check the references given. We will not dwell into details and introduce directly the relevant Riemann surface.

Let $\Delta_{k}, k=1, \ldots, m$, be a system of bounded intervals of the real line such that $\Delta_{k} \cap \Delta_{k+1}=\emptyset, k=1, \ldots, m-1$. Consider the $(m+1)$-sheeted Riemann surface

$$
\mathcal{R}=\overline{\bigcup_{k=0}^{m} \mathcal{R}_{k}},
$$

formed by the consecutively "glued" sheets

$$
\mathcal{R}_{0}:=\overline{\mathbb{C}} \backslash \Delta_{1}, \quad \mathcal{R}_{k}:=\overline{\mathbb{C}} \backslash\left(\Delta_{k} \cup \Delta_{k+1}\right), k=1, \ldots, m-1, \quad \mathcal{R}_{m}=\overline{\mathbb{C}} \backslash \Delta_{m},
$$

where the upper and lower banks of the slits of two neighboring sheets are identified. In fact, this describes $\mathcal{R}$ as a branched cover of $\overline{\mathbb{C}}$. Denote by $\pi$ the corresponding projection. Notice that $\mathcal{R}$ is a compact Riemann surface of genus 0 ; therefore, it is conformally equivalent to $\overline{\mathbb{C}}$. Fix $l \in\{1, \ldots, m\}$. We are interested in the conformal representation $\psi^{(l)}$ of $\mathcal{R}$ onto $\overline{\mathbb{C}}$ such that

$$
\psi^{(l)}(w)=w+\mathcal{O}(1), w \rightarrow \infty^{(l)}, \quad \psi^{(l)}(w)=C_{1} / w+\mathcal{O}\left(1 / w^{2}\right), w \rightarrow \infty^{(0)},
$$

where $w^{(l)}$ denotes the point in the sheet $l$ which projects onto $w \in \overline{\mathbb{C}}$. When $m=1$ and $\Delta_{1}=[b-2 a, b+2 a], \varphi=\frac{1}{a} \psi^{(1)} \mid \mathcal{R}^{(1)}$.

In view of our motivation coming from the ratio asymptotic of multiple orthogonal polynomials of Nikishin systems of measures, we would like to find an explicit expression for $\psi^{(l)}$ or, at least, an algebraic equation characterizing it, in terms of the end points of the intervals $\Delta_{k}$. This problem seems to be very difficult and 
unsolvable in all its generality, because it requires the solution of high-order algebraic equations. For this reason, in the main part of the paper, we will restrict our attention to the case $m=2$, where we have been able to achieve our goals (see Theorem 3.1 below).

There is another way of looking at the Riemann surface $\mathcal{R}$. If $\psi: \mathcal{R} \rightarrow \overline{\mathbb{C}}$ is any conformal representation and $g: \overline{\mathbb{C}} \rightarrow \mathcal{R}$ is its inverse, then

$$
G:=\pi \circ g
$$

is a holomorphic function from the extended Riemann sphere onto itself and hence it is a rational function. Its degree (understood as the number of solutions of the equation $G(z)=w$ for a generic $w$ ) equals the number of sheets of the branched cover $\pi: \mathcal{R} \rightarrow \overline{\mathbb{C}}$, that is, $m+1$. The end points of the intervals $\Delta_{k}$ determine, in fact, the values of $G$ at its critical points, where $G^{\prime}(z)=0$. Therefore, we are interested in determining a rational function $G$ of a given degree by its critical values and, possibly, some additional topological characteristics of the branched cover it defines.

We remark that a related problem of classifying rational functions with real critical values has been studied recently by various authors; see [10] and the references therein. It also turns out that our problem is related to a problem of determining the coefficients of a special Schwarz-Christoffel mapping of the upper half-plane onto a special Riemann surface; see $₫ 4$ below.

The paper is divided as follows. In Section 2, we describe some general properties of the conformal representations of $\mathcal{R}$ onto $\overline{\mathbb{C}}$. Section 3 contains the statement of the main results of the paper, which are obtained for the case when $m=2$. Their proofs are carried out in Sections 4 and 5. The final Section 6 is devoted to the development of a numerical algorithm for solving the system (Syst), which in turn allows one to calculate the functions $\psi^{(1)}, \psi^{(2)}$ numerically (for $m=2$ ).

Acknowledgements. We would like to thank the referee for carefully reading the manuscript and for some helpful suggestions. We also express our gratitude to Daniel Estévez Sánchez, who helped us with the numerical calculations, using the MatLab system. His work was subsidized by a Grant for the Support to Study by the Autonomic Community of Madrid (2008).

\section{Some General properties}

Let $\psi, \Psi$ be any two conformal representations of $\mathcal{R}$ onto $\overline{\mathbb{C}}$. Then $\Psi \circ \psi^{-1}$ is an automorphism of $\overline{\mathbb{C}}$. Consequently, there exist constants $a, b, c, d$, with $a d-c b \neq 0$, such that

$$
\Psi(w)=\frac{a \psi(w)+b}{c \psi(w)+d} .
$$

Therefore, if we have found a conformal representation $\psi$, any other $\Psi$ can be expressed explicitly in terms of $\psi$ and the values of $\Psi$ at three distinct points (alternatively, in terms of the values of $\Psi$ at two points and its first derivative at one of the two points). For example (see (1.1)),

$$
\psi^{(k)}(w)=C_{2} \psi^{(l)}(w) /\left(\psi^{(l)}(w)-\psi^{(l)}\left(\infty^{(k)}\right)\right), \quad k, l \in\{1, \ldots, m\}, \quad k \neq l,
$$

where $C_{2} \neq 0$ is an appropriate constant so that the Laurent expansion of the right-hand side at $\infty^{(k)}$ has leading coefficient equal to 1. 
Let $\psi_{k}^{(l)}, k=0,1, \ldots, m$, denote the branches of $\psi^{(l)}$ corresponding to the different sheets of $\mathcal{R}$. The function $\psi^{(l)}$ is the solution of the algebraic equation

$$
\prod_{k=0}^{m}\left(z-\psi_{k}^{(l)}(w)\right)=z^{m+1}+\sum_{j=0}^{m} \alpha_{j}(w) z^{j}=0 .
$$

The coefficients $\alpha_{j}(w)$ are the so-called symmetric functions which are known to be entire functions on the complex plane and can be expressed in terms of the branches of $\psi^{(l)}$ through the Vieta relations.

By the definition of $\psi^{(l)}, \alpha_{0}(w)=(-1)^{m+1} \prod_{k=0}^{m+1} \psi_{k}^{(l)}(w)$ has no singularity on $\overline{\mathbb{C}}$; therefore, according to Liouville's theorem, it is constant. On the other hand, each $\alpha_{j}(w), j=1, \ldots, m$, has a simple pole at $\infty$; consequently, it is a polynomial of first degree. In (2.3), we can solve for $w$ and from what was said before we find that $w=g(z)=p(z) / q(z)=\left(\psi^{(l)}\right)^{-1}(z)$, where $p$ is a polynomial of degree $m+1$ and $q$ is a polynomial of degree $m$. The algebraic equation which defines $\mathcal{R}$ is irreducible; therefore, $(p, q) \equiv 1$. Otherwise, $\psi^{(l)}$ would satisfy a lower-order algebraic equation in $z$. The poles of $g$ (which are $\infty$ and the zeros of $q$ ) are the points $\psi^{(l)}\left(\infty^{(k)}\right), k=0, \ldots, m$. The zeros of $g$ (which are the zeros of $p$ ) are the points $\psi^{(l)}\left(0^{(k)}\right), k=0, \ldots, m$ (without loss of generality we can assume that $\left.0 \notin \bigcup_{k=0}^{m} \Delta_{k}\right)$. Since $\psi^{(l)}$ is single-valued, all these points are distinct. In particular, the zeros and poles of $g$ are simple.

It is easy to verify that

$$
\psi^{(l)}(w)=\overline{\psi^{(l)}(\bar{w})}, \quad w \in \mathcal{R} .
$$

Indeed, let $\phi(w):=\overline{\psi^{(l)}(\bar{w})} . \phi$ and $\psi^{(l)}$ have the same divisor; consequently, there exists a constant $C$ such that $\phi=C \psi^{(l)}$. Comparing the leading coefficients of the Laurent expansion of these functions at $\infty^{(l)}$, we conclude that $C=1$.

In terms of the branches of $\psi^{(l)}$, the symmetry formula (2.4) indicates that for each $k=0,1, \ldots, m$,

$$
\psi_{k}^{(l)}: \overline{\mathbb{R}} \backslash\left(\Delta_{k} \cup \Delta_{k+1}\right) \longrightarrow \overline{\mathbb{R}}
$$

$\left(\Delta_{0}=\Delta_{m+1}=\emptyset\right)$; therefore, the coefficients (in particular, the leading one) of the Laurent expansions at $\infty$ of the branches of $\psi^{(l)}$ are real numbers, and

$$
\psi_{k}^{(l)}\left(t_{ \pm}\right)=\overline{\psi_{k}^{(l)}\left(t_{\mp}\right)}=\overline{\psi_{k+1}^{(l)}\left(t_{ \pm}\right)}, \quad t \in \Delta_{k+1}
$$

(the second equality is due to continuity).

\section{The RESUlts FOR $m=2$}

Here we consider the problem of explicitly determining the functions $G$ and $\psi^{(l)}$ for the case of two intervals $\Delta_{1}, \Delta_{2}$. Using an affine transformation, if necessary, we can assume that $\Delta_{1}=[-\mu,-1]$ and $\Delta_{2}=[1, \lambda]$, where $\lambda, \mu>1$.

Theorem 3.1. The functions $\psi^{(1)}$ and $\psi^{(2)}$ can be computed as follows:

$$
\psi^{(1)}=\frac{1}{H(a)}\left(1+G^{-1}\right), \quad \psi^{(2)}=\frac{A}{2 H(a)} \frac{1+G^{-1}}{1-G^{-1}},
$$

where $G(z):=H(z) / H(a)$, 


$$
\begin{gathered}
H(z)=h+z+\frac{A z}{1-z}+\frac{B z}{1+z}, \\
h=\frac{1}{4}(a+\alpha)\left(2 a \alpha-\frac{(a-\alpha)^{2}}{1-a \alpha}\right),
\end{gathered}
$$

$$
A=\frac{1}{4}(1-\beta)(1-\alpha)(1-a)(1-b), \quad B=\frac{1}{4}(1+\beta)(1+\alpha)(1+a)(1+b),
$$

$\beta$ and $b$ are the solutions of the equation

$$
x^{2}+(a+\alpha) x+\frac{(a-\alpha)^{2}}{1-a \alpha}-3=0,
$$

verifying $\beta<-1, b>1$, and $\alpha$ and $a$ are the unique solutions of the algebraic system

(Syst) $\left\{\begin{array}{l}2(a+\alpha)(3-a \alpha-a-\alpha)(3-a \alpha+a+\alpha)+(\lambda-\mu)(a-\alpha)^{3}=0, \\ (\lambda+\mu)^{2}(a-\alpha)^{6}=4(3+a \alpha)^{3}(1-a \alpha)(2+a+\alpha)(2-a-\alpha),\end{array}\right.$

verifying $-1<\alpha<a<1$.

Since $H^{-1}(w)$ is the solution of the cubic equation

$$
z^{3}-(w+A-B-h) z^{2}-(1+A+B) z+w-h=0,
$$

the functions $\psi^{(1)}$ and $\psi^{(2)}$ can be computed explicitly if we know $\alpha$ and $a$.

As follows from the proof, the rational function $G$, given by this theorem, is given alternatively by (1.2), where $g$ is one of the conformal homeomorphisms of $\overline{\mathbb{C}}$ onto $\mathcal{R}$. As part of the proof of Theorem 3.1 we will also obtain that $G$ and the real numbers $\beta, \alpha, a, b$ constitute a unique solution of the following system of relations:

$$
\left\{\begin{array}{l}
G=\frac{P}{Q}, \quad \operatorname{deg} P=3, \operatorname{deg} Q=2, \\
-1 \text { and } 1 \text { are poles of } G, \\
\lim _{z \rightarrow \infty} \frac{G(z)}{z}>0 \\
\beta<-1<\alpha<a<1<b \\
G^{\prime}(\beta)=G^{\prime}(\alpha)=G^{\prime}(a)=G^{\prime}(b)=0 \\
-\mu=G(\beta), \quad \lambda=G(b) \\
-G(\alpha)=G(a)=1 .
\end{array}\right.
$$

Theorem 3.2. Consider the following subsets of $\mathbb{R}^{2}$ :

$$
\mathfrak{A}=\{(a, \alpha):-1 \leq a<\alpha \leq 1\}, \quad \Omega=\{(\lambda, \mu): \lambda \geq 1, \mu \geq 1\} .
$$

Then system (Syst) defines a one-to-one correspondence between these sets. Moreover, this mapping transforms the set $\{(a, \alpha):-1<a<\alpha<1\}$ onto the interior of $\Omega$, the sides $\{a=-1\},\{\alpha=1\}$ of the triangle $\mathfrak{A}$ onto the rays $\{\lambda=1\},\{\mu=1\} \subset \Omega$, respectively, and the vertex $\left(a_{0}, \alpha_{0}\right)=(-1,1)$ of $\mathfrak{A}$ onto the point $(1,1) \in \Omega$.

If $\Delta_{1}$ and $\Delta_{2}$ have the same Euclidean length, then $\mu=\lambda$, and we obtain a very simple result.

Theorem 3.3. If $\lambda=\mu$, then

$$
\psi^{(1)}=\frac{1+a^{2}}{2 a^{3}}\left(G^{-1}+1\right), \quad \psi^{(2)}=\frac{\left(1-a^{2}\right)^{2}}{8 a^{3}} \frac{G^{-1}+1}{G^{-1}-1},
$$


where $G(z):=H(z) / H(a)$,

$$
H(z)=z-\frac{\left(1-a^{2}\right)^{2} z}{\left(1+a^{2}\right)\left(1-z^{2}\right)},
$$

and $a$ is the unique solution on the interval $(0,1)$ of the biquartic equation

$$
a^{8}+\left(16 \lambda^{2}-8\right) a^{6}+18 a^{4}-27=0 .
$$

In this case, $H^{-1}(w)$ is the solution of the cubic equation

$$
z^{3}-w z^{2}+\frac{a^{4}-3 a^{2}}{1+a^{2}} z+w=0 .
$$

\section{Proof of Theorem 3.1}

4.1. A geometric definition of $G$. Suppose intervals $\Delta_{1}=[-\mu,-1]$ and $\Delta_{2}=$ $[1, \lambda]$ are given. Then there exists a conformal homeomorphism $g: \overline{\mathbb{C}} \longrightarrow \mathcal{R}$ such that $G:=\pi \circ g: \overline{\mathbb{C}} \longrightarrow \overline{\mathbb{C}}$ is a rational function $G=P / Q$ with real coefficients, $\operatorname{deg} P=3$ and $\operatorname{deg} Q=2$. In fact, $g$ can be constructed as follows. Consider a conformal map $g$ from the closed upper half-plane $\overline{\mathbb{H}}$ onto the simply connected set

$$
\mathcal{R}^{+}:=\left\{\zeta \in \mathcal{R}_{0}: \Im \pi(\zeta) \leq 0\right\} \cup\left\{\zeta \in \mathcal{R}_{1}: \Im \pi(\zeta) \geq 0\right\} \cup\left\{\zeta \in \mathcal{R}_{2}: \Im \pi(\zeta) \leq 0\right\} .
$$

Notice that $\mathcal{R}^{+}$is "one half" of $\mathcal{R}$ and $g(\overline{\mathbb{R}})$ coincides with the boundary of $\mathcal{R}^{+}$. We can choose $g$ so that for some $\beta<z_{1}<\alpha<a<z_{2}<b$ we have $g((-\infty, \beta])=$ $(-\infty,-\mu]^{(1)}, g\left(\left[\beta, z_{1}\right]\right)=(-\infty,-\mu]^{(0)}, g\left(\left[z_{1}, \alpha\right]\right)=[-1, \infty)^{(0)}, g([\alpha, a])=[-1,1]^{(1)}$, $g\left(\left[a, z_{2}\right]\right)=(-\infty, 1]^{(2)}, g\left(\left[z_{2}, b\right]\right)=[\lambda, \infty)^{(2)}$ and $g([b, \infty))=[\lambda, \infty)^{(1)}$.

By Schwarz's reflection principle, we can extend $g$ to a conformal map (which we denote also by $g$ ) from $\mathbb{C}$ into $\mathcal{R}$, which is symmetric with respect to the real line. Then $G:=\pi \circ g: \overline{\mathbb{C}} \longrightarrow \overline{\mathbb{C}}$ is holomorphic; therefore, it is a rational function $G=P / Q$, where $P$ and $Q$ have real coefficients, since $G$ maps $\overline{\mathbb{R}}$ into $\overline{\mathbb{R}}$. As $G$ has degree 3 and has three poles $\left(z_{1}, z_{2}, \infty\right)$, then a fortiori $\operatorname{deg} P=3$ and $\operatorname{deg} Q=2$.

$G$ has two finite poles and four critical points on the real line. Taking $G\left(d_{1} z+d_{2}\right)$ (with $d_{1}>0, d_{2} \in \mathbb{R}$ ) instead of $G(z)$, if necessary, without loss of generality we can assume that the poles of $G$ are -1 and 1 . These normalizations define $G$ uniquely.

The critical points of $G$ satisfy

$$
\beta<-1<\alpha<a<1<b .
$$

Moreover, $G$ increases on $(-\infty, \beta),(\alpha, a),(b, \infty)$, and decreases on $(\beta,-1),(-1, \alpha)$, $(a, 1),(1, b)$. Therefore, $G(\alpha)<G(a)$; we also have $-\mu=G(\beta)<G(\alpha)=-1$ and $1=G(a)<G(b)=\lambda$.

It follows that $G$ satisfies all the relations (3.11).

This way of constructing $G$ shows that it is in fact a kind of Schwarz-Christoffel mapping from $\overline{\mathbb{H}}$ onto the Riemann surface $\mathcal{R}^{+}$, which may be viewed as an unbounded polygon, whose angles are all equal to $2 \pi$.

4.2. An auxiliary rational function $H$. In order to compute $G$, let us consider the rational function

$$
H(z):=c G(z)=c \frac{P(z)}{Q(z)}, \quad \operatorname{deg} P=3, \operatorname{deg} Q=2,
$$

where $c$ is a positive constant such that $\lim _{z \rightarrow \infty} H(z) / z=1$. 
Then, the requirements (3.11) on $G$ are equivalent to the following conditions on the rational function $H$ :

$$
\left\{\begin{array}{l}
H=\frac{P_{1}}{Q}, \quad \operatorname{deg} P_{1}=3, \operatorname{deg} Q=2, \\
-1 \text { and } 1 \text { are poles of } H \\
\mu=H(\beta) / H(\alpha), \quad \lambda=H(b) / H(a), \quad H(\alpha)=-H(a), \\
\beta<-1<\alpha<a<1<b \\
H^{\prime}(\beta)=H^{\prime}(\alpha)=H^{\prime}(a)=H^{\prime}(b)=0 \\
\lim _{z \rightarrow \infty} H(z) / z=1 .
\end{array}\right.
$$

Since -1 and 1 are simple poles of $H$, we get from the two last equations in (4.13) the equalities:

$$
H^{\prime}(z)=\frac{(z-\beta)(z-\alpha)(z-a)(z-b)}{\left(z^{2}-1\right)^{2}}=1+\frac{A}{(z-1)^{2}}+\frac{B}{(z+1)^{2}} .
$$

Therefore,

$$
H(z)=h+\int_{0}^{z} \frac{(\zeta-\beta)(\zeta-\alpha)(\zeta-a)(\zeta-b)}{\left(\zeta^{2}-1\right)^{2}} d \zeta=h+z+\frac{A z}{1-z}+\frac{B z}{1+z},
$$

where $h=H(0)$, so that (3.7) holds.

4.3. The deduction of system (Syst). We are going to prove equations (3.8)(3.10) and the validity of (Syst). In order to do that, we will express $\beta, b, h, A$ and $B$ in terms of $\alpha$ and $a$ and write down the equations in $\alpha, a$. The choice of $\alpha$ and $a$ as unknown variables seems to be the best one from the numerical point of view, because they are bounded $(-1<\alpha<a<1)$.

From the equation for $H^{\prime}(z)$ we deduce that

$$
(z-\beta)(z-\alpha)(z-a)(z-b)=\left(z^{2}-1\right)^{2}+A(z+1)^{2}+B(z-1)^{2} .
$$

Replacing $z=1$ and $z=-1$ in (4.14), we obtain

$$
4 A=(1-\beta)(1-\alpha)(1-a)(1-b), \quad 4 B=(1+\beta)(1+\alpha)(1+a)(1+b) .
$$

Identifying the coefficients in (4.14), it follows that

$$
\begin{aligned}
& -\beta-\alpha-a-b=0, \\
& \beta \alpha+\beta a+\beta b+\alpha a+\alpha b+a b=-2+A+B, \\
& -\beta \alpha a-\beta \alpha b-\beta a b-\alpha a b=2 A-2 B, \\
& \beta \alpha a b=1+A+B .
\end{aligned}
$$

Using (4.17) and (4.19), we get

$$
\alpha(\beta+b)+a(\beta+b)+\beta b+\alpha a=-3+\beta \alpha a b .
$$

Relation (4.16) gives $\beta+b=-(\alpha+a)$, and we deduce that

$$
\beta b=\frac{a^{2}+\alpha^{2}+a \alpha-3}{1-a \alpha}=-3+\frac{(a-\alpha)^{2}}{1-a \alpha} .
$$

Therefore, $\beta$ and $b$ can be obtained from $a$ and $\alpha$ as the solutions of the equation

$$
x^{2}+(a+\alpha) x+\frac{(a-\alpha)^{2}}{1-a \alpha}-3=0 .
$$


Since

$$
H(z)=h+z+\frac{A z}{1-z}+\frac{B z}{1+z},
$$

the condition $H(\alpha)=-H(a)$ means that

$$
\begin{aligned}
& 4 h+4 \alpha+\alpha(1-\beta)(1-a)(1-b)+\alpha(1+\beta)(1+a)(1+b) \\
= & -4 h-4 a-a(1-\beta)(1-\alpha)(1-b)-a(1+\beta)(1+\alpha)(1+b) .
\end{aligned}
$$

Using that $\beta+b=-(\alpha+a)$, we express $h$ in terms of $a$ and $\alpha$ :

$$
4 h=(a+\alpha)(2 a \alpha-3-\beta b)=(a+\alpha)\left(2 a \alpha-\frac{(a-\alpha)^{2}}{1-a \alpha}\right) .
$$

We are going to make use of the equations

$$
\left\{\begin{array}{l}
\lambda H(a)=H(b) \\
\mu H(a)=-H(\beta)
\end{array}\right.
$$

Since $H(\beta)<0<H(b)$, these equations are equivalent to the following ones:

$$
\left\{\begin{array}{l}
\lambda H(\beta)+\mu H(b)=0 \\
(\lambda+\mu) H(a)=H(b)-H(\beta) .
\end{array}\right.
$$

We have

$$
\begin{aligned}
2 H(a) & =2 h+2 a+\frac{a}{2}(1-\beta)(1-\alpha)(1-b)+\frac{a}{2}(1+\beta)(1+\alpha)(1+b) \\
& =2 h+a(3-\alpha(a+\alpha)+b \beta), \\
2 H(b) & =2 h+b \beta(a+\alpha)+b(3+a \alpha), \\
2 H(\beta) & =2 h+b \beta(a+\alpha)+\beta(3+a \alpha) .
\end{aligned}
$$

So we get from (4.26) that

$$
\left\{\begin{array}{l}
(\lambda+\mu)(2 h+b \beta(a+\alpha))+(\lambda \beta+\mu b)(3+a \alpha)=0, \\
(\lambda+\mu)(2 h+a(3-\alpha(a+\alpha)+b \beta))=(b-\beta)(3+a \alpha) .
\end{array}\right.
$$

Since $b$ and $\beta$ are the solutions of the equation $x^{2}+(a+\alpha) x+b \beta=0$, we have

$$
b=\frac{-(a+\alpha)+\sqrt{(a+\alpha)^{2}-4 b \beta}}{2} \quad \text { and } \quad \beta=\frac{-(a+\alpha)-\sqrt{(a+\alpha)^{2}-4 b \beta}}{2} \text {. }
$$

From this, we deduce that

$$
b-\beta=\sqrt{(a+\alpha)^{2}-4 b \beta}
$$

and

$$
\lambda \beta+\mu b=-\frac{\lambda+\mu}{2}(a+\alpha)+\frac{\mu-\lambda}{2} \sqrt{(a+\alpha)^{2}-4 b \beta}
$$

(one could, in fact, express here $b \beta$ in terms of $a$ and $\alpha$; see (4.21)). These last two equations and (4.21) imply that (4.27) is equivalent to:

$$
\left\{\begin{array}{l}
(\lambda+\mu)\left(4 h+\left(2 \frac{(a-\alpha)^{2}}{1-a \alpha}-6\right)(a+\alpha)\right) \\
\quad+\left(-(\lambda+\mu)(a+\alpha)+(\mu-\lambda) \sqrt{(a+\alpha)^{2}-4 b \beta}\right)(3+a \alpha)=0 \\
(\lambda+\mu)\left(2 h+a\left(-\alpha(a+\alpha)+\frac{(a-\alpha)^{2}}{1-a \alpha}\right)\right)=(3+a \alpha) \sqrt{(a+\alpha)^{2}-4 b \beta} .
\end{array}\right.
$$


After replacing $4 h$ by its value, given in (4.24), we get

$4 h+\left(2 \frac{(a-\alpha)^{2}}{1-a \alpha}-6\right)(a+\alpha)-(a+\alpha)(3+a \alpha)=(a+\alpha)\left(-9+a \alpha+\frac{(a-\alpha)^{2}}{1-a \alpha}\right)$

and

$$
2 H(a)=2 h+a\left(-\alpha(a+\alpha)+\frac{(a-\alpha)^{2}}{1-a \alpha}\right)=\frac{(a-\alpha)^{3}}{2(1-a \alpha)} .
$$

Then, (4.28) is equivalent to

$$
\left\{\begin{array}{l}
(\lambda+\mu)(a+\alpha)\left(-9+a \alpha+\frac{(a-\alpha)^{2}}{1-a \alpha}\right)+(\mu-\lambda) \sqrt{(a+\alpha)^{2}-4 b \beta}(3+a \alpha)=0 \\
(\lambda+\mu) \frac{(a-\alpha)^{3}}{1-a \alpha}=2(3+a \alpha) \sqrt{(a+\alpha)^{2}-4 b \beta} .
\end{array}\right.
$$

Substituting the second equation in (4.30) into the first one, we obtain an equivalent system:

$$
\left\{\begin{array}{l}
2(a+\alpha)\left(-9+a \alpha+\frac{(a-\alpha)^{2}}{1-a \alpha}\right)+(\mu-\lambda) \frac{(a-\alpha)^{3}}{1-a \alpha}=0, \\
(\lambda+\mu) \frac{(a-\alpha)^{3}}{1-a \alpha}=2(3+a \alpha) \sqrt{(a+\alpha)^{2}-4 b \beta} .
\end{array}\right.
$$

The first equation in (4.31) is equivalent to the first equation in (Syst). Since $-1<$ $\alpha<a<1$, both terms in the second equation in (4.31) are positive. Therefore, this equation is equivalent to

$$
(\lambda+\mu)^{2} \frac{(a-\alpha)^{6}}{(1-a \alpha)^{2}}=4(3+a \alpha)^{2}\left((a+\alpha)^{2}+12-4 \frac{(a-\alpha)^{2}}{1-a \alpha}\right),
$$

and hence to the second equation in (Syst).

We conclude that the functions $G$ and $H$ are necessarily given by the formulas in Theorem 3.1, where $a$ and $\alpha$ are some solution of (Syst). The uniqueness of the solution will be checked in the next subsection.

4.4. The reverse arguments. First we need some technical lemmas.

Lemma 4.1. Let $t_{1}, t_{2}$ be the solutions of the real equation $t^{2}+p t+q=0$, with $\Re t_{1} \leq \Re t_{2}$. Then $t_{1}<-1$ and $t_{2}>1$ if and only if $1+|p|<-q$.

Proof. The proofs are obvious because the condition $t_{1}<-1$ and $t_{2}>1$ is equivalent to $f(-1)<0$ and $f(1)<0$, where $f(t):=t^{2}+p t+q$.

Lemma 4.2. Let $a, \alpha$ be any real numbers such that $-1<\alpha<a<1$. Then the solutions $\beta, b$ of the equation

$$
x^{2}+(a+\alpha) x+\frac{(a-\alpha)^{2}}{1-a \alpha}-3=0
$$

are real. Assuming that $\beta \leq b$, one has $\beta<-1, b>1$.

Proof. By Lemma 4.1, it is sufficient to show that

$$
1+|a+\alpha|<3-\frac{(a-\alpha)^{2}}{1-a \alpha},
$$

whenever $-1<\alpha \leq a<1$. By symmetry, it suffices to verify that

$$
W(a, \alpha):=a+\alpha+\frac{(a-\alpha)^{2}}{1-a \alpha}<2 \quad \text { if }|\alpha| \leq a<1 .
$$


But this is a direct consequence of

$$
2-W(a, \alpha)=\frac{(1-a)(1-\alpha)(2+a+\alpha)}{1-a \alpha}>0 .
$$

Lemma 4.3. Suppose $\lambda>1, \mu>1$ are arbitrary real numbers. Let $\widetilde{G}$ be the unique rational function that satisfies (3.11), and define $\widetilde{H}$ from (4.12).

(i) There is at least one solution $(\alpha, a)$ to the system (Syst) such that $-1<$ $\alpha<a<1$.

(ii) Given any such solution, define $\beta<-1, b>1$ from (3.10), $A, B, h$ from (3.9), (3.8), and then define the corresponding function $H$ from (3.7). Set $G(z)=H(z) / H(a)$. Then $G=\widetilde{G}, H=\widetilde{H}$.

Proof. Part (i) has already been checked in subsection 4.3 .

Now assume that $\alpha, a, b, \beta, H, G$ have been constructed as in (ii). We show that

$$
H^{\prime}(z)=1+\frac{A}{(z-1)^{2}}+\frac{B}{(z+1)^{2}}=\frac{(z-\beta)(z-\alpha)(z-a)(z-b)}{\left(z^{2}-1\right)^{2}} .
$$

It suffices to check equations (4.16)-(4.19).

We have (4.16) from the definition of $\beta$ and $b$. This definition also gives (4.21), which implies (4.20). This last equation is the difference of (4.17) and (4.19). The definitions of $A$ and $B$ give

$$
2 A+2 B=1+\beta \alpha+\beta a+\beta b+\alpha a+\alpha b+a b+\beta \alpha a b,
$$

and this equation is the sum of (4.17) and (4.19). Therefore, we can also obtain the equations (4.17) and (4.19). The definitions of $A$ and $B$ also gives (4.18):

$$
\begin{aligned}
-2 A+2 B & =\beta+\alpha+a+b+\beta \alpha a+\beta \alpha b+\beta a b+\alpha a b \\
& =\beta \alpha a+\beta \alpha b+\beta a b+\alpha a b .
\end{aligned}
$$

Consequently, we have proved (4.32).

The definition of $h$ and the formulas

$$
\beta+b=-a-\alpha, \quad \beta b=\frac{(a-\alpha)^{2}}{1-a \alpha}-3
$$

give (4.23), which is equivalent to $H(\alpha)=-H(a)$.

We can reverse the arguments given in Section 4.1 to show the implications (Syst) $\Rightarrow(4.30 \Rightarrow$ (4.26). We also see that (4.29) holds, which, together with the second equation in system (4.26), implies that $H(a) \neq 0, H(b) \neq H(\beta)$. Therefore, we can deduce (4.25) from (4.26). Hence all the conditions (4.13) hold.

Now, we can assert that $H$ increases on $(-\infty, \beta),(\alpha, a),(b, \infty)$ and decreases on $(\beta,-1),(-1, \alpha),(a, 1),(1, b)$. Since $H$ increases on $(\alpha, a)$, we have $H(\alpha)<H(a)$, but $H(\alpha)=-H(a)$, so we deduce that $H(a)>0$.

Then $H^{\prime}(\beta)=H^{\prime}(\alpha)=H^{\prime}(a)=H^{\prime}(b)=0, H(\overline{\mathbb{R}})=\overline{\mathbb{R}}$ and $\lim _{z \rightarrow \infty} H(z) / z=1$. Furthermore, $H$ has just three poles, namely $-1,1$ and $\infty$, and they are simple.

The function $G(z)=H(z) / H(a)$ satisfies $G(\beta)=-\mu, G(\alpha)=-1, G(a)=1$ and $G(b)=\lambda$. We also have $G^{\prime}(\beta)=G^{\prime}(\alpha)=G^{\prime}(a)=G^{\prime}(b)=0$ and $G(\overline{\mathbb{R}})=\overline{\mathbb{R}}$.

Since $H(a)>0$, the function $G$ increases on $(-\infty, \beta),(\alpha, a),(b, \infty)$ and decreases on $(\beta,-1),(-1, \alpha),(a, 1),(1, b)$.

$G$ is a rational function of degree 3 ; therefore, we conclude that $G$ gives rise to a conformal map $g$ of $\overline{\mathbb{C}}$ onto a Riemann surface $\mathcal{R}^{*}$ with three sheets $\left(\mathcal{R}^{*}, \mathcal{R}_{1}^{*}, \mathcal{R}_{2}^{*}\right)$, so that $G=\pi \circ g$, where $\pi: \mathcal{R}^{*} \rightarrow \overline{\mathbb{C}}$ is the canonical projection. The set of branch 
points of $\mathcal{R}^{*}$ is $\{G(\beta), G(\alpha), G(a), G(b)\}=\{-\mu,-1,1, \lambda\}$. All these branch points have order two, because $G^{\prime}$ has simple zeros on $\beta, \alpha, a, b$.

Since $\mathcal{R}^{*}=\mathcal{R}^{*} \cup \mathcal{R}_{1}^{*} \cup \mathcal{R}_{2}^{*}$ is connected and the branch points have order two, there is some $\mathcal{R}_{j}^{*}$ (for instance, $\mathcal{R}_{1}^{*}$ ) with two cuts and $\mathcal{R}^{*}, \mathcal{R}_{2}^{*}$ have just one cut. By the monotonicity properties of $G$, we can deduce that $G(\infty)=\infty^{(1)}, G(-1)=\infty^{(0)}$, $G(1)=\infty^{(2)}$, and that $G(\overline{\mathbb{H}})=\left(\mathcal{R}^{*}\right)^{+}$, where $\left(\mathcal{R}^{*}\right)^{+}$is the union of one half of each $\mathcal{R}_{j}^{*}(j=0,1,2)$. We also have $G(\overline{\mathbb{R}})=\partial\left(\mathcal{R}^{*}\right)^{+}$. Every point of $\overline{\mathbb{R}} \backslash([-\mu,-1] \cup[1, \lambda])$ has 3 preimages by $G$ in $\overline{\mathbb{R}}$, and every point of $(-\mu,-1) \cup(1, \lambda)$ has one preimage by $G$ in $\overline{\mathbb{R}}$. Since $\left(\mathcal{R}^{*}\right)^{+}$is the union of one half of each $\mathcal{R}_{j}^{*}$ and it is connected, the cuts are $(-\mu,-1) \cup(1, \lambda)$. Consequently, $\mathcal{R}^{*}=\mathcal{R}$.

Therefore, $G^{-1} \circ \widetilde{G}$ is a conformal map from $\overline{\mathbb{C}}$ onto $\overline{\mathbb{C}}$, and it must be a Möbius transformation. Since $G(\infty)=\widetilde{G}(\infty)=\infty^{(1)}, G(-1)=\widetilde{G}(-1)=\infty^{(0)}, G(1)=$ $\widetilde{G}(1)=\infty^{(2)}$, the Möbius map $G^{-1} \circ \widetilde{G}$ fixes the points $\infty,-1$ and 1. It follows that $G^{-1} \circ \widetilde{G}$ is the identity map, and therefore $G=\widetilde{G}$.

Corollary 4.4. The solution ( $\alpha, a)$ to system (Syst) with the properties $-1<\alpha<$ $a<1$ is unique.

Proof. Indeed, as shown above, any solution $(\alpha, a)$ of this system gives rise to a function $G$, which coincides with $\widetilde{G}$. Denote the parameters that correspond to $\widetilde{G}$ by $\tilde{\alpha}, \tilde{a}$, etc. Then $\alpha=\tilde{\alpha}$ and $a=\tilde{a}$ (because they are the only two critical points of $G$ and $\widetilde{G}$ on the interval $(-1,1))$.

Since $G(z)=H(z) / H(a)$, we obtain that $G^{-1}(w)=H^{-1}(H(a) w)$. We have

$$
w=H(z)=\frac{(h+z)\left(1-z^{2}\right)+A(1+z) z+B(1-z) z}{1-z^{2}},
$$

or equivalently,

$$
z^{3}-(w+A-B-h) z^{2}-(1+A+B) z+w-h=0 .
$$

Observe that (4.33) allows us to obtain an explicit expression for $H^{-1}$ (and then for $\psi^{(1)}$ and $\left.\psi^{(2)}\right)$, once we have solved (Syst).

We now compute $\psi^{(1)}$ and $\psi^{(2)}$ in terms of $G$. Recall that $\psi^{(1)}$ and $\psi^{(2)}$ are determined by

$$
\begin{array}{ll}
\psi^{(1)}(w)=w+\mathcal{O}(1), w \rightarrow \infty^{(1)}, & \psi^{(1)}\left(\infty^{(0)}\right)=0, \\
\psi^{(2)}(w)=w+\mathcal{O}(1), w \rightarrow \infty^{(2)}, & \psi^{(2)}\left(\infty^{(0)}\right)=0 .
\end{array}
$$

Note that $G^{-1}\left(\infty^{(0)}\right)=-1, G^{-1}\left(\infty^{(1)}\right)=\infty$ and $G^{-1}\left(\infty^{(2)}\right)=1$. Hence, $\psi^{(1)}=c_{1}\left(1+G^{-1}\right)$ for some constant $c_{1}$. In order to calculate the constant $c_{1}$, notice that

$$
\begin{aligned}
& H(z)=z+\mathcal{O}(1), \quad z \rightarrow \infty, \\
& H^{-1}(w)=w+\mathcal{O}(1), \quad w \rightarrow \infty^{(1)}, \\
& G^{-1}(w)=H(a) w+\mathcal{O}(1), \quad w \rightarrow \infty^{(1)}, \\
& w+\mathcal{O}(1)=c_{1} H(a) w+\mathcal{O}(1), \quad w \rightarrow \infty^{(1)},
\end{aligned}
$$

and then

$$
c_{1}=\frac{1}{H(a)} .
$$


We also have $\psi^{(2)}=c_{2}\left(1+G^{-1}\right) /\left(1-G^{-1}\right)$ for some constant $c_{2}$. In order to compute the constant $c_{2}$, let us expand the left-hand side of (4.33) in powers of $(z-1)$. One gets that (4.33) is equivalent to

$$
\begin{aligned}
& (z-1)^{3}+(3-w-A+B+h)(z-1)^{2}+(2-2 w-3 A+B+2 h)(z-1)-2 A=0 \\
& \frac{(z-1)^{2}}{w}+\frac{(3-w-A+B+h)(z-1)}{w}+\frac{2-2 w-3 A+B+2 h}{w}-\frac{2 A}{w(z-1)}=0 .
\end{aligned}
$$

Since $H^{-1}\left(\infty^{(2)}\right)=1$, we have that

$$
\lim _{w \rightarrow \infty^{(2)}} \frac{2 A}{w\left(1-H^{-1}(w)\right)}=\lim _{w \rightarrow \infty^{(2)}} \frac{2 A}{w(1-z)}=2,
$$

or, equivalently,

$$
\begin{aligned}
\lim _{w \rightarrow \infty^{(2)}} \frac{2}{w\left(1-G^{-1}(w)\right)} & =\lim _{w \rightarrow \infty^{(2)}} \frac{2}{w\left(1-H^{-1}(H(a) w)\right)} \\
& =\lim _{w \rightarrow \infty^{(2)}} \frac{2 H(a)}{w\left(1-H^{-1}(w)\right)}=\frac{2 H(a)}{A} .
\end{aligned}
$$

Recall that

$$
\begin{aligned}
1=\lim _{w \rightarrow \infty^{(2)}} \frac{\psi^{(2)}(w)}{w} & =c_{2} \lim _{w \rightarrow \infty^{(2)}} \frac{1+G^{-1}(w)}{w\left(1-G^{-1}(w)\right)} \\
& =c_{2} \lim _{w \rightarrow \infty^{(2)}} \frac{2}{w\left(1-G^{-1}(w)\right)}=c_{2} \frac{2 H(a)}{A}
\end{aligned}
$$

and this gives $c_{2}=A /(2 H(a))$. This finishes the proof of Theorem 3.1

\section{Proofs of Theorems 3.2 and 3.3}

Proof of Theorem 3.2 . We associate with any point $(\lambda, \mu) \in \mathbb{R}^{2}$ the point $(u, v)$, where

$$
u=\lambda-\mu, \quad v=(\lambda+\mu)^{2} .
$$

In terms of $u, v$, (Syst) may be rewritten as

$$
\left\{\begin{array}{l}
u=-\frac{2(a+\alpha)(3-a \alpha-a-\alpha)(3-a \alpha+a+\alpha)}{(a-\alpha)^{3}} \\
v=4 \frac{(3+a \alpha)^{3}(1-a \alpha)(2+a+\alpha)(2-a-\alpha)}{(a-\alpha)^{6}}
\end{array}\right.
$$

One can check that these equations imply that 1

$$
\begin{aligned}
& v-(2-u)^{2}=16 \frac{\left(a^{2}-1\right)\left(\alpha^{2}+2 a \alpha-3\right)^{3}}{(a-\alpha)^{6}} \\
& v-(2+u)^{2}=16 \frac{\left(\alpha^{2}-1\right)\left(a^{2}+2 a \alpha-3\right)^{3}}{(a-\alpha)^{6}}
\end{aligned}
$$

Notice that $\alpha^{2}+2 a \alpha-3<0$ for all $(a, \alpha) \in(-1,1) \times(-1,1)$. It also follows that $\alpha^{2}+2 a \alpha-3 \leq 0$ for all $(a, \alpha) \in[-1,1] \times[-1,1]$.

\footnotetext{
${ }^{1}$ We found these formulas by using the package Maple of symbolic calculations.
} 
Take any point $(a, \alpha) \in \mathfrak{A}$. By (5.36), the corresponding pair $(u, v)$, given by (5.35), satisfies $v \geq(2-u)^{2}, v \geq(2+u)^{2}$. Hence $v \geq 0, \sqrt{v} \geq 2-u$, and $\sqrt{v} \geq 2+u$. Put

$$
\lambda=\frac{1}{2}(u+\sqrt{v}), \quad \mu=\frac{1}{2}(\sqrt{v}-u) ;
$$

then $\lambda, \mu \geq 1$. By examining (5.34), one gets that the pair $(\lambda, \mu)$ is the unique solution of (Syst) that belongs to $\Omega$. By repeating the same arguments with strict inequalities, one also sees that $\lambda>1, \mu>1$ whenever $(a, \alpha) \in \operatorname{int} \mathfrak{A}$. The assertions of the theorem concerning the images in $\Omega$ of the sets $\{\alpha=-1\},\{a=1\}$, and $\{\alpha=-1, a=1\}$ follow easily from (5.36).

It was proved already in the previous section that for any $(\lambda, \mu) \in \operatorname{int} \Omega$, (Syst) has a unique solution $(a, \alpha) \in \operatorname{int} \mathfrak{A}$. This finishes the proof of the theorem.

Proof of Theorem 3.3. Assume that the intervals $[-\mu,-1]$ and $[1, \lambda]$ are symmetric, i.e. $\lambda=\mu$. Then, taking into account that $(3-a \alpha-a-\alpha)(3-a \alpha+a+\alpha)>0$, we deduce from the first equation in (Syst) that $\alpha=-a$. Hence (4.24) gives $h=0$. By (4.22), it follows that $\beta$ and $b$ are the solutions of the equation

$$
x^{2}=3-\frac{4 a^{2}}{1+a^{2}}=\frac{3-a^{2}}{1+a^{2}} .
$$

Consequently, $\beta=-b$. Hence, by (4.15), $B=A=-\frac{\left(1-a^{2}\right)^{2}}{2\left(1+a^{2}\right)}$. Therefore,

$$
H(z)=z-\frac{\left(1-a^{2}\right)^{2} z}{\left(1+a^{2}\right)\left(1-z^{2}\right)}, \quad H(a)=\frac{2 a^{3}}{1+a^{2}},
$$

and

$$
\psi^{(1)}=\frac{1+a^{2}}{2 a^{3}}\left(G^{-1}+1\right), \quad \psi^{(2)}=\frac{\left(1-a^{2}\right)^{2}}{8 a^{3}} \frac{G^{-1}+1}{G^{-1}-1} .
$$

Consequently, $H$ is an odd function, and the second equation in (Syst) yields

$$
16 \lambda^{2} a^{6}=\left(1+a^{2}\right)\left(3-a^{2}\right)^{3}=27-18 a^{4}+8 a^{6}-a^{8} .
$$

We have obtained that $a$ is solution of

$$
a^{8}+\left(16 \lambda^{2}-8\right) a^{6}+18 a^{4}-27=0 .
$$

In this simpler case, we can check directly that there is exactly one solution of (5.38) on the interval $(0,1)$. In fact, the function $u(t)=t^{4}+\left(16 \lambda^{2}-8\right) t^{3}+18 t^{2}-27$ verifies $u(0)=-27<0, u(1)=16 \lambda^{2}-16>0$, and $u^{\prime}(t)=4 t^{3}+3\left(16 \lambda^{2}-8\right) t^{2}+36 t>36 t>0$ for every $t \in(0,1)$. Furthermore, since (5.38) is a biquartic equation, it can be solved by radicals.

\section{Numerical RESOlution of THE NONLINEAR SYSTEM}

The purpose of this section is to show that the problem of solving numerically the algebraic system (Syst) is a simple task, both from the theoretical and practical points of view.

Let us use the vector notation: $A=(\alpha, a) \in \operatorname{int} \mathfrak{A}$ and $L=(\lambda, \mu) \in \operatorname{int} \Omega$. By Theorem 3.2, (Syst) defines uniquely a map

$$
F: A \mapsto L .
$$


We suggest to use a continuation method for solving this equation numerically, which is a variation of methods described, for instance, in [4], Section 4.4.2. Notice that these methods go back to the treatise 8 by H. Poincaré on celestial mechanics.

Recall that the Newton method for solving equation $F(A)=\widehat{L}$ with the starting point $A_{s t}$ consists in the following (see, for instance, 4], Section 4.2). Fix a small parameter $\sigma>0$ and define iteratively $A^{(0)}=A_{s t} ; A^{(n+1)}=A^{(n)}-$ $F^{\prime}\left(A^{(n)}\right)^{-1} F\left(A^{(n)}\right), n \geq 0$. This iterative process finishes when $\left|F\left(A^{(n)}\right)-\widehat{L}\right|<\sigma$, and the last point $A_{\text {fin }}:=A^{(n)}$ is taken for an approximate solution of equation $F(A)=\widehat{L}$.

In the continuation scheme, we apply the Newton algorithm several times. Given a point $L_{*} \in$ int $\Omega$, we wish to find a good approximation for a solution $A_{*} \in$ int $\mathfrak{A}$ of the equation $F\left(A_{*}\right)=L_{*}$. The method goes as follows.

Part 1. Choose an initial approximation $A_{0}$ of the solution. We do this by solving the equation $F\left(A_{0}\right)=L_{0}$, where $L_{0}=\left(\lambda_{*}+\mu_{*}, \lambda_{*}+\mu_{*}\right) / 2$ is a symmetric vector, and by applying Theorem 3.3 .

Part 2. Choose a large integer $n>0$, and divide the interval $\left[L_{0}, L_{*}\right] \subset \Omega$ into $n$ equal subintervals by division points $L_{0}, L_{1}, \ldots, L_{n}=L_{*}$. So, we set $L_{k}=$ $\left((n-k) L_{0}+k L_{*}\right) / n$.

Part 3. Let the parameter $\sigma>0$ be fixed. The calculation is performed in $n$ steps, applying the Newton method $n$ times.

At the $k$ th step $(1 \leq k \leq n)$, we find an approximate solution $A_{f i n}^{(k)}$ of the equation $F(A)=L_{k}$ by running the above-described Newton method with the starting point $A_{s t}^{(k)}=A_{\text {fin }}^{(k-1)}$, which is available from the $(k-1)$ th step. If $k=1$, then we put $A_{s t}^{(1)}=A_{0}$, where $A_{0}$ is the value found from Part 1.

Once all $n$ steps of Part 3 are performed successfully, one takes $A_{f i n}^{(n)}$ for an approximation of the solution $A_{*}$ of the equation $F\left(A_{*}\right)=L_{*}$. One has, in fact: $F\left(A_{\text {fin }}^{(n)}\right) \approx L_{n}=L_{*}$.

The applicability of this method for our concrete function $F$ is justified by the following theorem. We will formulate it in a more general setting.

Theorem 6.1. Let $\widetilde{\mathfrak{A}}$ and $\widetilde{\Omega}$ be open subsets of $\mathbb{R}^{d}$, and suppose that $\widetilde{\mathfrak{A}}$ is connected and $\widetilde{\Omega}$ is convex. Let $F$ be a $\mathcal{C}^{2}$ homeomorphism from $\widetilde{\mathfrak{A}}$ to $\widetilde{\Omega}$ such that $\operatorname{det} F^{\prime} \neq 0$ in $\widetilde{\mathfrak{A}}$. Then the above continuation scheme for the Newton method is numerically feasible in the following sense.

Let a point $L_{*} \in \operatorname{int} \Omega$ and a starting point $L_{0}$ be given. Let $A_{*} \in \widetilde{\mathfrak{A}}$ be the (unique) solution of the equation $F(A)=L_{*}$. Then for any $\varepsilon>0$ there exist a $\sigma_{0}>0$ and an integer $N_{0} \geq 1$ such that for any $n>N_{0}$ and any $\sigma<\sigma_{0}$, the Newton method stops at each of the $n$ steps of Part 3 of the algorithm, and the approximate solution $A_{\text {fin }}^{(n)}=A_{\text {fin }}^{(n)}(\sigma)$ obtained satisfies $\left|A_{\text {fin }}^{(n)}-A_{*}\right|<\varepsilon$.

Because of the topological assumption on $F$, the situation is very simple. The feasibility of the method is closely related with the continuation property for $F$; see [7. Section 5.3.

It seems that this theorem is a variation of classical results, known to specialists. Since the authors were unable to find an exact reference, a sketch of the proof is included. 
Proof of Theorem 6.1. Denote by $B(q, r)$ the open ball centered at a point $q \in \mathbb{R}^{d}$ of radius $r$. We apply Theorem 4.10 from [4]. It gives sufficient quantitative conditions for convergence of the Newton method, which imply, in particular, the following. Suppose $\widehat{A} \in \widetilde{\mathfrak{A}}$, and let $\widehat{L}=F(\widehat{A}) \in \widetilde{\Omega}$. Then, by the assumption, $\operatorname{det} F^{\prime}(\widehat{A}) \neq 0$. It follows that there exists $\delta>0$ with the following property.

(*) Given any $\varepsilon>0$, there exists $\sigma_{0}>0$ such that to any $\sigma<\sigma_{0}$ there corresponds an integer $M(\sigma)$ satisfying the following: the Newton method for solving equation $F(A)=\widehat{L}$ with any starting point $A_{s t} \in B(\widehat{A}, \delta)$ stops after at most $M(\sigma)$ steps, and the approximate solution $A_{\text {fin }}(\sigma)$ obtained satisfies $\left|A_{\text {fin }}(\sigma)-\widehat{A}\right|<\varepsilon$.

Next, the pre-image $F^{-1}\left(\left[L_{0}, L_{*}\right]\right)$ is compact; hence the infimum of $\left|\operatorname{det} F^{\prime}\right|$ on this set is positive. It follows that the above condition $\left(^{*}\right)$ takes place uniformly for all pairs of points $(\widehat{A}, \widehat{L}) \in \widetilde{\mathfrak{A}} \times \widetilde{\Omega}$ such that $F(\widehat{A})=\widehat{L}$ and $\widehat{L} \in\left[L_{0}, L_{*}\right]$.

Fix $L_{0}, L_{*}$, and fix some $\delta$ such that $\left(^{*}\right)$ holds for all pairs $(\widehat{A}, \widehat{L})$ as above. Take any $\varepsilon>0$, and let us prove that the conclusions of the theorem hold for this $\varepsilon$. We can assume that $\varepsilon<\delta / 2$. Let us find the corresponding $\sigma_{0}$ such that $(*)$ holds. There exists a positive $\rho$ such that $\left|A^{\prime}-A^{\prime \prime}\right|<\varepsilon$ whenever $F\left(A^{\prime}\right), F\left(A^{\prime \prime}\right) \in\left[L_{0}, L_{*}\right]$ and $\left|F\left(A^{\prime}\right)-F\left(A^{\prime \prime}\right)\right|<\rho$. Let $N_{0}$ be any integer such that $h N_{0}>\left|L_{*}-L_{0}\right|$. We claim that the assertion of the theorem holds for these $\sigma_{0}$ and $N_{0}$.

Indeed, take any $n \geq N_{0}$, and put (as above) $L_{k}=\left((n-k) L_{0}+k L_{*}\right) / n$ and $A_{k}=F^{-a}\left(L_{k}\right)$. Then $\left|A_{k}-A_{k+1}\right|<\varepsilon$ for all $k$. Consider the properties:

$$
(\boldsymbol{\Gamma})_{k} \quad\left|A_{s t}^{(k)}-A_{k}\right|<\delta ; \quad(\boldsymbol{\Delta})_{k} \quad\left|A_{f i n}^{(k)}-A_{k}\right|<\varepsilon .
$$

Since $A_{s t}^{(k)}=A_{0},(\boldsymbol{\Gamma})_{1}$ holds. Next, for any $k,(\Gamma)_{k}$ implies $(\Delta)_{k}$, due to $(*)$, and $(\Delta)_{k}$ implies $(\Gamma)_{k+1}$, because

$$
\left|A_{s t}^{(k+1)}-A_{k+1}\right|=\left|A_{\text {fin }}^{(k)}-A_{k+1}\right| \leq\left|A_{\text {fin }}^{(k)}-A_{k}\right|+\left|A_{k}-A_{k+1}\right|<2 \varepsilon<\delta .
$$

By induction, we obtain that $(\Gamma)_{k}$ and $(\Delta)_{k}$ hold for all $k$. In particular,

$$
\left|A_{\text {fin }}^{(n)}-A_{n}\right|=\left|A_{\text {fin }}^{(n)}(\sigma)-A_{*}\right|<\varepsilon .
$$

Let us return to our particular function $F$, defined by system (Syst). It follows from Theorem 3.2 and the explicit formulas (5.35), (5.37) that $F: \operatorname{int} \mathfrak{A} \rightarrow \operatorname{int} \Omega$ is a $\mathcal{C}^{\infty}$ smooth homeomorphism. Put $\widetilde{\mathfrak{A}}=\operatorname{int} \mathfrak{A}$ and $\widetilde{\Omega}=\operatorname{int} \Omega$. In order to prove that the above Theorem 6.1 applies to $F$, it only remains to check that $\operatorname{det} F^{\prime}$ does not vanish in int $\mathfrak{A}$. This follows from an explicit calculation, using formulas (5.35), (5.37), that represents $F$ as a composition map. One has

$$
\operatorname{det} F^{\prime}=\operatorname{det} \frac{\partial(\lambda, \mu)}{\partial(a, \alpha)}=\operatorname{det} \frac{\partial(\lambda, \mu)}{\partial(u, v)} \cdot \operatorname{det} \frac{\partial(u, v)}{\partial(a, \alpha)} .
$$

Then, det $\frac{\partial(\lambda, \mu)}{\partial(u, v)}=1 /\left(4 v^{1 / 2}\right)>0$, because $v=v(a, \alpha)>0$ in int $\mathfrak{A}$. At last, (5.35) implies that

$$
\operatorname{det} \frac{\partial(u, v)}{\partial(a, \alpha)}=-2^{7}(a-\alpha)^{-10}\left(a^{2}+2 a \alpha-3\right)^{2}(3+a \alpha)^{2}\left(\alpha^{2}+2 a \alpha-3\right)^{2}
$$


(we have applied the Maple package here). As we noted in the proof of Theorem 3.2. $a^{2}+2 a \alpha-3<0$ and $\alpha^{2}+2 a \alpha-3<0$ for all $(a, \alpha) \in$ int $\mathfrak{A}$. Therefore $\operatorname{det} \frac{\partial(u, v)}{\partial(a, \alpha)}<0$ and $\operatorname{det} F^{\prime}(A)<0$ for all $A=(a, \alpha) \in \operatorname{int} \mathfrak{A}$.

In Table 1, we reproduce some numerical results of the implementation of the above method.

TABLE 1. Numerical results

\begin{tabular}{|c|c|c|c|c|c|}
\hline$\lambda$ & $\mu$ & $\beta$ & $\alpha$ & $a$ & $b$ \\
\hline 1.01 & 1.10 & -1.02433457 & -0.97566543 & 0.99756619 & 1.00243381 \\
\hline 1.01 & 1.50 & -1.11120778 & -0.88879220 & 0.99776894 & 1.00223104 \\
\hline 1.01 & 2.00 & -1.20162987 & -0.79837009 & 0.99796298 & 1.00203698 \\
\hline 1.01 & 5.00 & -1.53512664 & -0.46487320 & 0.99855894 & 1.00144090 \\
\hline 1.01 & 10.00 & -1.80340393 & -0.19659585 & 0.99893546 & 1.00106432 \\
\hline 1.01 & 20.00 & -2.05587223 & 0.05587246 & 0.99922942 & 1.00077035 \\
\hline 1.01 & 50.00 & -2.33815403 & 0.33815423 & 0.99950547 & 1.00049433 \\
\hline 1.01 & 100.00 & -2.50602945 & 0.50602961 & 0.99964857 & 1.00035127 \\
\hline 1.10 & 1.50 & -1.10904414 & -0.89095444 & 0.97812791 & 1.02187067 \\
\hline 1.10 & 2.00 & -1.19803860 & -0.80195744 & 0.97999986 & 1.01999618 \\
\hline 1.10 & 5.00 & -1.52833535 & -0.47164952 & 0.98579364 & 1.01419123 \\
\hline 1.10 & 10.00 & -1.79584373 & -0.20413551 & 0.98948341 & 1.01049582 \\
\hline 1.10 & 20.00 & -2.04866217 & 0.04868414 & 0.99237698 & 1.00760105 \\
\hline 1.10 & 50.00 & -2.33227827 & 0.33229733 & 0.99510249 & 1.00487845 \\
\hline 1.10 & 100.00 & -2.50132594 & 0.50134152 & 0.99651797 & 1.00346645 \\
\hline 1.50 & 2.00 & -1.18411168 & -0.81581475 & 0.90730105 & 1.09262537 \\
\hline 1.50 & $\overline{5.00}$ & -1.50111605 & -0.49858460 & 0.93306652 & 1.06663413 \\
\hline 1.50 & 10.00 & -1.76499059 & -0.23458419 & 0.95001591 & 1.04955887 \\
\hline 1.50 & 20.00 & -2.01887210 & 0.01933234 & 0.96355662 & 1.03598315 \\
\hline 1.50 & 50.00 & -2.30775501 & 0.30816158 & 0.97647774 & 1.02311569 \\
\hline 1.50 & 100.00 & -2.48160429 & 0.48193938 & 0.98324158 & 1.01642333 \\
\hline 2.00 & 5.00 & -1.47250352 & -0.52657019 & 0.87477665 & 1.12429705 \\
\hline 2.00 & 10.00 & -1.73155665 & -0.26707834 & 0.90556526 & 1.09306973 \\
\hline 2.00 & 20.00 & -1.98589261 & -0.01259181 & 0.93068264 & 1.06780179 \\
\hline 2.00 & 50.00 & -2.28012155 & 0.28148839 & 0.95501596 & 1.04361720 \\
\hline 2.00 & 100.00 & -2.45919974 & 0.46033681 & 0.96787312 & 1.03098981 \\
\hline 5.00 & 10.00 & -1.59758021 & -0.39392722 & 0.70769711 & 1.28381032 \\
\hline 5.00 & 20.00 & -1.84539761 & -0.14406679 & 0.77852683 & 1.21093757 \\
\hline 5.00 & 50.00 & -2.15584295 & 0.16630302 & 0.85234419 & 1.13719574 \\
\hline 5.00 & 100.00 & -2.35580726 & 0.36490617 & 0.89323258 & 1.09766851 \\
\hline 10.00 & 20.00 & -1.70949675 & -0.26791189 & 0.61012964 & 1.36727900 \\
\hline 10.00 & 50.00 & -2.02314506 & 0.04811583 & 0.73131282 & 1.24371641 \\
\hline 10.00 & 100.00 & -2.23983688 & 0.26275325 & 0.80256032 & 1.17452331 \\
\hline 20.00 & 50.00 & -1.86312347 & -0.09255784 & 0.56794501 & 1.38773629 \\
\hline 20.00 & 100.00 & -2.09037160 & 0.13418100 & 0.67474116 & 1.28144945 \\
\hline 50.00 & 100.00 & -1.85498816 & -0.06912394 & 0.44519539 & 1.47891671 \\
\hline
\end{tabular}




\section{REFERENCES}

[1] A.I. Aptekarev, G. López Lagomasino, and I.A. Rocha, Asymptotic behavier of the ratio of Hermite-Padé polynomials for Nikishin systems, Mat. Sb. 196 (2005), 1089-1107. MR2188362 (2006h:41040)

[2] A.I. Aptekarev, V. Kalyagin, G. López Lagomasino, and I.A. Rocha, On the limit behavior of recurrence coefficients for multiple orthogonal polynomials, J. of Approx. Theory 139 (2006), 346-370. MR2220045 (2007a:42048)

[3] S. A. Denisov, On Rakhmanov's theorem for Jacobi matrices, Proc. Amer. Math. Soc. 132 (2004), 847-852. MR2019964 (2005h:47060)

[4] P. Deuflhard and A. Hofmann, Numerical analysis in modern scientific computing. An introduction (Second edition). Texts in Applied Mathematics, 43. Springer, New York, 2003. MR1949263(2003i:65001)

[5] A. López García and G. López Lagomasino, Ratio asymptotic of Hermite-Padé orthogonal polynomials for Nikishin systems. II, Advances in Math. 218 (2008), 1081-1106. MR2419380

[6] P. Nevai, Orthogonal Polynomials, Mem. Amer. Math. Soc., no. 213, Providence, R. I., 1979. MR.519926 (80k:42025)

[7] J.M. Ortega and W. C. Rheinboldt, Iterative solutions of nonlinear equations in several variables, Acad. Press, N.Y.-London, 1970. MR0273810 (42:8686)

[8] H. Poincaré, Les Méthodes Nouvelles de la Mécanique Celeste. Gauthier-Villars, Paris, 1892.

[9] E. A. Rakhmanov, On asymptotic properties of polynomials orthogonal on the unit circle with weights not satisfying Szegö's condition, Math. USSR Sb. 58 (1987), 149-167. MR0854969 (88b:42033)

[10] B. Shapiro and A. Vainstein, Counting real rational functions with all real critical values, Moscow Math. J. 3 no. 2 (2003), 647-659. MR2025277 (2006d:26024)

Department of Mathematics, Universidad Carlos III de Madrid, 28911 Leganes, Spain

E-mail address: lago@math.uc3m.es

Department of Mathematics, Universidad Carlos III de Madrid, 28911 Leganes, Spain

E-mail address: dompes@math.uc3m.es

Department of Mathematics, Universidad Carlos III de Madrid, 28911 Leganes, Spain

E-mail address: jomaro@math.uc3m.es

Department of Mathematics, Universidad, Autónoma de Madrid and Instituto de Ciencias, Mathemáticas (CSIC-UAM-UC3M-UCM), Madrid, Spain

E-mail address: dmitry.yakubovich@uam.es 